\title{
THERMODYNAMIC PROPERTIES OF $D$-WAVE PAIRED SUPERCONDUCTORS
}

\author{
R. Gonczarek, M. Mulak and W. Kumala \\ Institute of Physics, Wrocław University of Technology \\ Wybrzeże Wyspiańskiego 27, 50-370 Wrocław, Poland \\ (Received May 29, 1998; revised version July 14, 1998; \\ in final form December 31, 1998)
}

The $D$-wave paired Fermi system is considered in the frame of generalized gap equation obtained in a BCS-like approach. It is shown that the thermodynamic functions of a system, as the thermodynamic potential, entropy and heat capacity differences between the normal and superconducting state are precisely defined functionals of the energy gap as a function of temperature. The derived formulas are identical as those obtained for $S$-wave paired Fermi system. It states that the developed formalism can be applied, in practice, to investigation of real superconductors in which a singlet state is realized. Some numerical results illustrating prospects and capabilities of the presented formalism are given for chosen structures of the order parameter.

PACS numbers: $74.20 .-\mathrm{z}$

\section{Introduction}

In the present investigations of heavy fermion, organic or cuprate superconductors the $D$-wave state is favored as the best candidate for really occurring paired state (cf. [1]). The possible and permitted ground states in $D$-wave paired Fermi system (superconductor) have been theoretically examined, in the frame of the weak coupling theory, by Anderson and Morel [2].

The aim of this paper is to study some thermodynamic properties of such $D$-wave states, based on their results. We are going to show that the general thermodynamic relations, and some final results, elaborated previously in the frame of our parametric formalism for $S$-wave paired Fermi system [3-6], can be now successfully transformed to the case of $D$-wave pairing. The basis for the parametric formalism is the generalized energy gap equation obtained in a BCS-like approach $[3,4]$, and now respectively modified.

The new results are derived strictly analytically and what is worth to emphasize, they should allow one to broaden the class of superconductors which can be investigated in the frame of the presented set of parametric equations. Now, they could be applied to real superconductors in which a singlet state is realized, although we do not know exactly which type of paired states occur. 


\section{General relations}

\subsection{The energy gap equation}

In the paper, we focus our interest on such theoretical models of superconductor, for which the energy gap equation can be written in the following, general form:

$$
\hat{\Delta}(\hat{p})=5 g_{2}\left\langle P_{2}\left(\hat{p} \cdot \hat{p}^{\prime}\right) \Phi\left(\hat{p}^{\prime}\right) \hat{\Delta}\left(\hat{p}^{\prime}\right)\right\rangle
$$

It is convenient to refer the above notation to the paper [7]. Let us describe more precisely the origin of Eq. (1) and all introduced above quantities. Analogously as in [7] we start from the BCS equation for arbitrary pairing interaction, but now taking into account only pure $D$-wave pairing of a system, i.e. only $L=2$, omitting the term $V_{0} P_{0}$ responsible for creation of $S$-wave pairing. Afterwards, in the starting equation the summation over momentum space is replaced by the integration over spherical angles $(\theta, \varphi)$, where the notation is used

$$
\langle\ldots\rangle=\frac{1}{4 \pi} \int_{0}^{2 \pi} \mathrm{d} \varphi \int_{0}^{\pi} \mathrm{d} \theta \sin \theta \ldots
$$

which finally leads to Eq. (1).

Because only one Legendre harmonic $L=2$ is considered, we deal now only with the dimensionless coupling parameter $g_{2}$, which in general is equal to

$$
g_{L}=\frac{V_{L}}{2 L+1} N(0)
$$

where $V_{L}$ are coefficients of expansion of the pairing potential in series of Legendre polynomials $P_{2}\left(\hat{p} \cdot \hat{p}^{\prime}\right)$. Moreover

$$
P_{2}\left(\hat{p} \cdot \hat{p}^{\prime}\right)=\frac{4 \pi}{5} \sum_{m=-2}^{m=2} Y_{2 m}(\theta, \varphi) Y_{2 m}^{*}\left(\theta^{\prime}, \varphi^{\prime}\right)
$$

where $\hat{p}$ and $\hat{p}^{\prime}$ denote the direction of the quasiparticle (electron) momentum on the Fermi surface and $N(0)$ is the density of states per spin and per unit volume on the Fermi surface.

The functional $\Phi$ in Eq. (1) is now of the form

$$
\Phi(\hat{p})=\int_{0}^{\omega_{\mathrm{D}}} \frac{\mathrm{d} \xi}{\sqrt{\xi^{2}+|\hat{\Delta}(\hat{p})|^{2}}} F\left(\frac{\xi}{2 T}, \frac{|\hat{\Delta}(\hat{p})|}{2 T}\right),
$$

where the function $F\left(l_{1}, l_{2}\right)$ reduces to the standard tanh for the classical BCS model. The function $F$, as a crucial generalization of the BCS energy gap equation, was originally introduced in [3]. The properties of $F$ are analogous as for BCS case i.e. it is restricted and can be approximated, in the limiting cases of the arguments $l_{1}, l_{2}$, according to the relations

$$
\begin{aligned}
& F\left(0, l_{2}\right) \sim l_{2} \quad \text { if } \quad l_{2} \rightarrow 0, \\
& F\left(l_{1}, l_{2}\right) \equiv F_{\infty} \quad \text { if } \quad l_{1} \quad \text { or } \quad l_{2} \text { tends to infinity. }
\end{aligned}
$$

Let us note that for the classical BCS system $F_{\infty}=1$. In the third chapter of the paper we restrict our considerations and introduce the specific form of $F$ for $D$-wave paired superconductor. Now we still keep its general form. 
Let us discuss briefly the physical background of Eq. (1). It represents the wide class of models of superconductor which can be obtained from BCS-type Hamiltonian after including additional corrections of Fermi liquid interactions in the system. The functional form of $\Phi(\hat{p})$ constitutes the generalization of the weak coupling as well as Hubbard type approaches established in the literature (cf. [8-10]). Moreover, such a structure of $\Phi$ allows one to transform the integral in (4) into the more convenient form for further considerations in the next chapter.

Finally, it is worth to emphasize that the definition of (4) admits into considerations a non-parabolic shape of the kinetic energy of quasiparticles. In such a case, in the vicinity of Fermi level, the density of states is not anymore considered as a constant, equal to $N(0)$, but depends on $\xi$. Consequently, we deal with the particle-hole asymmetry on the Fermi level. As an example, in the case of BCS-like $D$-wave paired superconductor we may define the function $F$, which corresponds to such particle-hole asymmetry, in the following way:

$$
F\left(\frac{\xi}{2 T}, \frac{|\hat{\Delta}(\hat{p})|}{2 T}\right)=I(\xi) \tanh \left(\frac{\sqrt{\xi^{2}+|\hat{\Delta}(\hat{p})|^{2}}}{2 T}\right),
$$

where

$$
I(\xi)=\frac{N(\xi)+N(-\xi)}{2 N(0)}
$$

describes directly the degree of particle-hole asymmetry, for the particular shape of $N(\xi)$. Let us note that exactly on the Fermi surface $I(0)=1$. Furthermore, if $\xi$ is large, the relation $I(\xi) \rightarrow F_{\infty}$ must be fulfilled, according to the properties of $F$ (see (5)) and because then $\tanh \rightarrow 1$. We emphasize, that the form of $F$ in Eq. (6) has only an exemplary character. The mentioned particle-hole asymmetry has the interesting physical consequence, namely, if electronic density of states is an oscillating function just in the Fermi surface vicinity, the critical temperature of the superconductor is strongly enhanced, as it was shown in [11].

In the studied case of $D$-wave pairing we state that

$$
\hat{\Delta}(\hat{p})=\Delta(T) \cdot D(\hat{p})
$$

where $\Delta(T)$ defines the temperature dependence of the energy gap. In turn, $D(\hat{p})$ characterizes the spherical angles dependence of the pairing and is a mixture of spherical harmonics $Y_{2 m}$ defined as

$$
D(\hat{p})=\sum_{m=-2}^{m=2} d_{m} Y_{2 m}(\theta, \varphi) .
$$

Above we assume that the equilibrium state of a superconducting phase can be constructed as a superposition of the eigenvalue states $Y_{2 m}$. According to (7) and the normalization condition $\left\langle Y_{2 m}^{*} Y_{2 m}\right\rangle=(1 / 4 \pi) \delta_{m n}$, the coefficients $d_{m}$ must fulfill the relation

$$
\sum_{m=-2}^{m=2}\left|d_{m}\right|^{2}=4 \pi
$$


Physically, $d_{m}$ determine the contribution of particular eigenvalue states. Moreover, because

$$
\left\langle\hat{\Delta} \hat{\Delta}^{\dagger}\right\rangle=\Delta^{2}(T)\left\langle\left|D^{2}\right|\right\rangle=\Delta^{2}(T),
$$

it must be also $\left\langle\left|D^{2}\right|\right\rangle=1$.

Let us transform Eq. (1) to the more convenient form in further considerations. Employing Eq. (3), Eq. (7) and the condition (8) the energy gap equation (1) can be rewritten as a set of the equations

$$
\frac{d_{m}}{4 \pi g_{2}}=\left\langle Y_{2 m}^{*} D \Phi\right\rangle
$$

where $m=0, \pm 1, \pm 2$. However, using Eq. (7) once more, we can rebuild Eqs. (9) to the form

$$
\frac{1}{g_{2}}=\left\langle|D|^{2} \Phi\right\rangle \text {. }
$$

Note that the set of Eqs. (9) always have solutions if some equations disappear and others reduce to the equivalent form. It takes place, for example, when $D(\hat{p})$ is of the form [2]

$$
D(\hat{p})=d_{m} Y_{2, m}(\theta, \varphi) \quad \text { or } \quad D(\hat{p})=d_{m}\left[Y_{2, m}(\theta, \varphi) \pm Y_{2,-m}(\theta, \varphi)\right] .
$$

Then, non-vanishing equations reduce, respectively, to the forms

$$
\frac{1}{4 \pi g_{2}}=\left\langle\left|Y_{2 m}\right|^{2} \Phi\right\rangle \text { and } \frac{1}{4 \pi g_{2}}=\left\langle\left|Y_{2 m}^{*}\right|^{2} \Phi\right\rangle \pm\left\langle Y_{2 m}^{*} Y_{2,-m} \Phi\right\rangle,
$$

where we also employ the relation $Y_{2,-m}^{*}=(-1)^{m} Y_{2 m}$. However, in general case of Eq. (7) the set of Eqs. (9) becomes very complicated. Then it is more convenient to consider Eq. (10) and include other physical circumstances.

\subsection{Thermodynamic potential}

In order to consider the thermodynamic properties of the system we have to derive the formula for $\Delta \Omega$ which defines the thermodynamic potential difference between the $D$-wave paired and the normal state. Let us note that the differential of the $\Delta \Omega$ can be written in the form

$$
\mathrm{d} \Delta \Omega=-\frac{\mathrm{d} \Delta \Omega}{\mathrm{d} g_{2}} g_{2}^{2} \mathrm{~d} \frac{1}{g_{2}} .
$$

According to the well-known rules given in [12]

$$
\frac{\mathrm{d} \Delta \Omega}{\mathrm{d} g_{2}}=-5\left\langle\left\langle P_{2}\left(\hat{p} \hat{p}^{\prime}\right) \Phi(\hat{p}) \hat{\Delta}^{*}(\hat{p})\right\rangle_{\hat{p}} \Phi\left(\hat{p}^{\prime}\right) \hat{\Delta}\left(\hat{p}^{\prime}\right)\right\rangle_{\hat{p}^{\prime}} .
$$

Employing now Eq. (3) the above Eq. (13) may be put as

$$
\frac{\mathrm{d} \Delta \Omega}{\mathrm{d} g_{2}}=-\sum_{m=-2}^{m=2} 4 \pi\left|\left\langle Y_{2 m}^{*} \Phi \hat{\Delta}\right\rangle\right|^{2}=-4 \pi \Delta^{2}(T) \sum_{m=-2}^{m=2}\left|\left\langle Y_{2 m}^{*} \Phi D\right\rangle\right|^{2}
$$

and according to Eq. (9) we get

$$
\frac{\mathrm{d} \Delta \Omega}{\mathrm{d} g_{2}}=-\frac{\Delta^{2}}{g_{2}^{2}}
$$

where $\Delta \equiv \Delta(T)$. 
Hence, using Eq. (10) and changing the order of integration we have

$$
\Delta \Omega=N(0) \int_{0}^{\Delta} \Delta^{\prime 2} \frac{\mathrm{d}}{\mathrm{d} \Delta^{\prime}}\left\langle|D|^{2} \Phi\right\rangle \mathrm{d} \Delta^{\prime} .
$$

Including the symbol

$$
Y=\frac{\Delta}{T}{ }_{c}
$$

Eq. (16) can be rewritten in the equivalent form

$$
\Delta \Omega=N(0) T_{\mathrm{c}}^{2}\left\langle|D|^{2} \int_{0}^{Y}\left(Y^{\prime}\right)^{2} \frac{\mathrm{d} \Phi}{\mathrm{d} Y^{\prime}} \mathrm{d} Y^{\prime}\right\rangle .
$$

Now, introducing also the parameters

$$
u=\frac{\xi}{2 T}, \quad \tau=\frac{\Delta}{2 T}
$$

and integrating (4) by parts we get (cf. [3])

$$
\begin{aligned}
\Phi= & F_{\infty} \ln \frac{2 \omega_{\mathrm{D}}}{T_{\mathrm{c}}}-F_{\infty} \ln Y-F_{\infty} \ln |D| \\
& -\int_{0}^{\infty} \mathrm{d} u\left[\ln \left(u+\sqrt{u^{2}+\tau^{2}|D|^{2}}\right)-\ln (\tau|D|)\right] \frac{\partial}{\partial u} F(u, \tau|D|) .
\end{aligned}
$$

From Eq. (19) implies more formal and concise form of $\Phi$, namely

$$
\Phi=-F_{\infty} \ln Y+f(\tau,|D|)
$$

where we assume about $f$ that it is some analytical function. Therefore Eq. (10) may be reduced to

$$
\frac{1}{g_{2}}=-F_{\infty} \ln Y+\left\langle|D|^{2} f(\tau,|D|)\right\rangle \text {. }
$$

Now, employing Eqs. (20) and (21), Eq. (18) can be transformed into the crucial form (see Appendix and [3]).

$$
\frac{\Delta \Omega}{N(0) T_{\mathrm{c}}^{2} F_{\infty}}=-\frac{1}{2} Y^{2}(\tau)-8 X^{2}(\tau) \int_{0}^{\tau} \ln \frac{Y\left(\tau^{\prime}\right)}{Y(\tau)} \tau^{\prime} \mathrm{d} \tau^{\prime},
$$

where $X=T / T_{\mathrm{c}}$ and

$$
X(\tau)=\frac{1}{2 \tau} Y(\tau) .
$$

The relation (22) is identical with that obtained in [4] for $S$-wave paired Fermi system. Therefore all presented there formulas for the entropy, heat capacity, and energy gap are valid also in the case of $D$-wave paired system.

In order to derive $\Delta \Omega$ we have to know the form of $Y(\tau)$. To find $Y(\tau)$ we deal with Eq. (19), applying the same analytical procedures performed in details previously for $S$-wave pairing in [3]. Namely, we first investigate Eq. (19) in two characteristic limits $T \rightarrow 0$ and $T \rightarrow T_{\mathrm{c}}$ and using the properties of $F$ (see (5)) we get the relations between $\omega_{\mathrm{D}}, T_{\mathrm{c}}$, and $\Delta(0)$. Next we eliminate them from Eq. (19) and solve it with respect to $Y(\tau)$. We have 


$$
\begin{aligned}
Y(\tau) & =Y_{0} \exp \left[-\frac{1}{F_{\infty}}\left\langle|D|^{2} \int_{0}^{\infty}\left[\ln \left(u+\sqrt{u^{2}+\tau^{2}|D|^{2}}\right)-\ln (\tau|D|)\right]\right.\right. \\
& \left.\left.\times \frac{\partial}{\partial u} F(u, \tau|D|) \mathrm{d} u\right\rangle\right]
\end{aligned}
$$

where

$Y_{0}=\frac{\Delta(0)}{T_{\mathrm{c}}}=2 \exp \left[-\left\langle|D|^{2} \ln |D|\right\rangle\right] \exp \left[\frac{1}{F_{\infty}} \int_{0}^{\infty} \ln (2 u) \frac{\partial}{\partial u} F(u, 0) \mathrm{d} u\right]$.

\subsection{Entropy and heat capacity}

Let us write down the relations for some other basic thermodynamic quantities, which can be obtained from Eq. (22), now in the case of $D$-wave pairing, according to [4]. Applying the basic relations between entropy, heat capacity, and thermodynamic potential given in [12], we get from Eq. (22) the entropy difference between $D$-wave paired and normal state in the form

$$
\frac{\Delta S}{16 N(0) T_{\mathrm{c}} F_{\infty}}=X(\tau) \int_{0}^{\tau} \ln \frac{Y\left(\tau^{\prime}\right)}{Y(\tau)} \tau^{\prime} \mathrm{d} \tau^{\prime}
$$

and the heat capacity difference between $D$-wave paired and normal state, respectively as

$$
\frac{\Delta C}{16 N(0) T_{\mathrm{c}} F_{\infty}}=\frac{\Delta S}{16 N(0) T_{\mathrm{c}} F_{\infty}}-\frac{1}{8} Y(\tau) \frac{\partial Y(\tau)}{\partial X(\tau)} .
$$

Based on Eqs. (26), (27) and the fundamental thermodynamic relations between the entropy and heat capacity [12], we can express both of them as functionals of the energy gap in a function of temperature $Y(\tau)$. We have

$$
\frac{\Delta S}{2 N(0) T_{\mathrm{c}} F_{\infty}}=X \int_{X}^{1} \frac{Y^{2}\left(X^{\prime}\right)}{\left(X^{\prime}\right)^{3}} \mathrm{~d} X^{\prime}-\frac{1}{2} \frac{Y^{2}(X)}{X}
$$

and consequently

$$
\frac{\Delta C}{2 N(0) T_{\mathrm{c}} F_{\infty}}=X \int_{X}^{1} \frac{Y^{2}\left(X^{\prime}\right)-Y^{2}(X)}{\left(X^{\prime}\right)^{3}} \mathrm{~d} X^{\prime}-\frac{1}{2} X Y^{2}(X)-Y(X) \frac{\partial Y(X)}{\partial X} .
$$

Furthermore, it is worth to emphasize that the last Eq. (29) can be reversed. Hence the energy gap as a functional of the heat capacity can be written down as follows $[11]$ :

$$
Y(X)=\left\{Y^{2}(0)-\frac{1}{2 N(0) T_{\mathrm{c}} F_{\infty}} \int_{0}^{X}\left[\Delta C\left(X^{\prime}\right)-\int_{0}^{X^{\prime}} \frac{\Delta C\left(X^{\prime \prime}\right)}{X^{\prime \prime}} \mathrm{d} X^{\prime \prime}\right] \mathrm{d} X^{\prime}\right\}^{1 / 2}
$$

After putting in Eq. (30) $X=1$ we have $Y(1)=0$ and then one can find $Y(0)$, i.e. the reduced energy gap at $T=0$. Hence, we may compare it with theoretical values obtained for some $D$-wave or $S$-wave paired states.

Moreover, Eq. (29) allows us to state that $\Delta C$ as well as $C_{\mathbf{s}}$, in the limit of $T \rightarrow 0$, is a function of the form (cf. Appendix)

$$
\Delta C=-a X-b \frac{\partial Y(X)}{\partial X}
$$


where $a$ and $b$ are constants. Hence we conclude that $\partial Y(X) / \partial X$ must always tend to zero if only $T \rightarrow 0$. In other words, the energy gap as a function of temperature must have a plateau in the zero-temperature region. The last two relations. (30) and (31) are valid for any singlet paired superconductor.

\section{Results for BCS-like system}

In order to illustrate the developed general formalism, let us restrict now our considerations to the BCS-like model of $D$-wave paired superconductor. It allows one to obtain the analytical and numerical results for some chosen $D$-wave paired states. The function $F$ is now defined in the form [3]

$$
F(u, \tau \cdot|D|)=\tanh \sqrt{u^{2}+\tau^{2}|D|^{2}} .
$$

According to the paper of Anderson and Morel [2], the $D$-wave ground state configuration is a mixture of spherical harmonics $Y_{20}, Y_{22}$, and $Y_{2,-2}$. More precisely, the most favorable $D$-wave solution is in the form

$$
\frac{1}{\sqrt{2}} Y_{20}+\frac{1}{2}\left(Y_{22}-Y_{2,-2}\right)
$$

Taking the above result into account we postulate to investigate slightly more general case of a mixture of spherical harmonics

$$
\hat{\Delta}=\Delta_{\varepsilon}(T) \sqrt{4 \pi}\left[\varepsilon Y_{20}+\sqrt{\frac{1-\varepsilon^{2}}{2}}\left(Y_{22}-Y_{2,-2}\right)\right] \text {. }
$$

The parameter $\varepsilon$, which in [2] is equal to $1 / \sqrt{2}$, is now a real number from the interval $0 \leq \varepsilon \leq 1$ and entirely determines the form of the equilibrium state of the system, when the $D$-wave coupling is included. We admit also that now $\Delta(T) \equiv$ $\Delta_{\varepsilon}(T)$ can depend on the given state. After some calculations and developing the forms of $Y_{20}, Y_{22}, Y_{2,-2},|D|$ as a function of $\varepsilon$ reduces to the form

$$
|D|=\frac{\sqrt{5}}{2}\left[\varepsilon^{2}\left(3 x^{2}-1\right)^{2}+3\left(1-\varepsilon^{2}\right)\left(1-x^{2}\right)^{2} \sin ^{2} 2 \varphi\right]^{1 / 2}
$$

with $x=\cos \theta$. The relation (34) defines the angle dependence of the energy gap in the case under consideration.

For such chosen states the relation between $\Delta_{\varepsilon}(0)$ and $T_{c}$ (which now depends on $\varepsilon$ ) takes the form

$$
\frac{\Delta_{\varepsilon}(0)}{T_{\mathrm{c}}}=\pi \mathrm{e}^{-C} \exp \left[-\left\langle|D|^{2} \ln |D|\right\rangle\right],
$$

where Eq. (25) was employed with $F_{\infty}=1$ and the integral equal to $\ln \left(\pi / 2 \mathrm{e}^{C}\right)$ (see [3]). Let us note that for all $\varepsilon$

$$
\frac{\Delta_{\varepsilon}(0)}{T_{\mathrm{c}}}<\pi \mathrm{e}^{-C}=1.76
$$

where $\pi \mathrm{e}^{-C}=1.76$ is a ratio's value for standard BCS case.

Let us illustrate the obtained results graphically. The plot of the above characteristic ratio $\Delta_{\varepsilon}(0) / T_{\mathrm{c}}$ (divided by $\pi \mathrm{e}^{-C}$ ) as a function of $\varepsilon$, according to Eq. (35) and using Eq. (34) is depicted in Fig. 1. Note that Eq. (35) after integration over spherical angles depends only on $\varepsilon$. The maximum in the plot for $\varepsilon=1 / \sqrt{2} \approx 0.71$, 


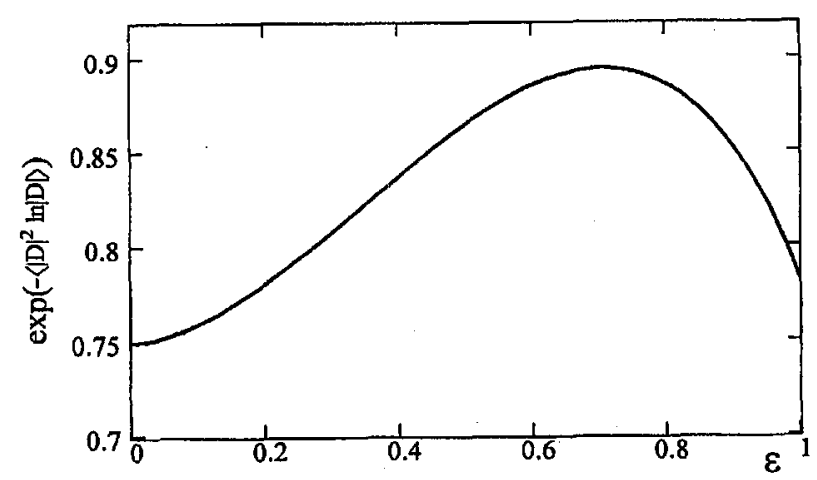

Fig. 1. The characteristic BCS ratio $\Delta_{\epsilon}(0) / T_{\mathrm{c}}$ divided by $\pi \mathrm{e}^{-C}$ as a function of $\varepsilon$ calculated numerically according to Eq. (35) using also Eq. (34); $\varepsilon$ is a real number $0 \leq$ $\varepsilon \leq 1$ and determines the form of the equilibrium state (mixture of eigenvalue states), see (33). The characteristic and extreme values of the ratio in the plot are the following: 0.75 for $\varepsilon=0,0.89$ (maximum) for $\varepsilon=1 / \sqrt{2}=0.71$ and 0.78 for $\varepsilon=1$. The maximum value of the ratio corresponds to the most favorable $D$-wave state (with $\varepsilon=1 / \sqrt{2}$ ) determined by Anderson and Morel in [2]. Note also that $\Delta_{\varepsilon} / T_{\mathrm{c}}<\pi \mathrm{e}^{-C}=1.76$ for all $\varepsilon$.

corresponds to the optimal $D$-wave ground state configuration discussed in [2]. At this point, the coupling of the Cooper pairs should be the strongest. For some other extreme values of the plot see description. Figure 1 is helpful in interpretation of Fig. 2 and Fig. 3, which are directly related to it. In turn, for all temperatures from 0 to $T_{c}$ we have

$$
\begin{aligned}
& \frac{\Delta_{\varepsilon}(T)}{T_{\mathrm{c}}}=\pi \mathrm{e}^{-C} \exp \left[-\left\langle|D|^{2} \ln |D|\right\rangle\right] \\
& \times \exp \left[-\left\langle|D|^{2} \int_{0}^{\infty}\left[\ln \left(u+\sqrt{u^{2}+\tau^{2}|D|^{2}}\right)-\ln (T|D|)\right]\right.\right. \\
& \left.\left.\times \frac{u}{\sqrt{u^{2}+\tau^{2}|D|^{2}}} \cosh ^{-2} \sqrt{u^{2}+\tau^{2}|D|^{2}} \mathrm{~d} u\right\rangle\right]
\end{aligned}
$$

and now, what is worth to emphasize,

$$
\frac{T(\tau)}{T_{\mathrm{c}}}=\frac{1}{2 \tau} \frac{\Delta_{\varepsilon}(\tau)}{T_{\mathrm{c}}}
$$

The above ratio given by (36) is plotted, for the exemplary set of $\varepsilon$ from Fig. 1 (see description), in Fig. 2. The curves in the left plot correspond to the values of $\varepsilon$ from the interval $0 \leq \varepsilon \leq 1 / \sqrt{2}$, i.e. from 0 to the maximum in Fig. 1 and in the right one, to some $\varepsilon$ from the interval $1 / \sqrt{2} \leq \varepsilon \leq 1$, i.e. from the maximum to 1 , respectively. This two separate pictures of Fig. 2 should help one to recognize the dynamic of changes of $\Delta(T)$ with $\varepsilon$ (i.e. the influence of $\varepsilon$ on the strength of Cooper pairing).

Based on Fig. 2 we can also approximate the relative change of the ratio $\Delta_{\varepsilon}(0) / T_{\mathrm{c}}$ with respect to $\varepsilon$. It does not exceed $10 \%$. 


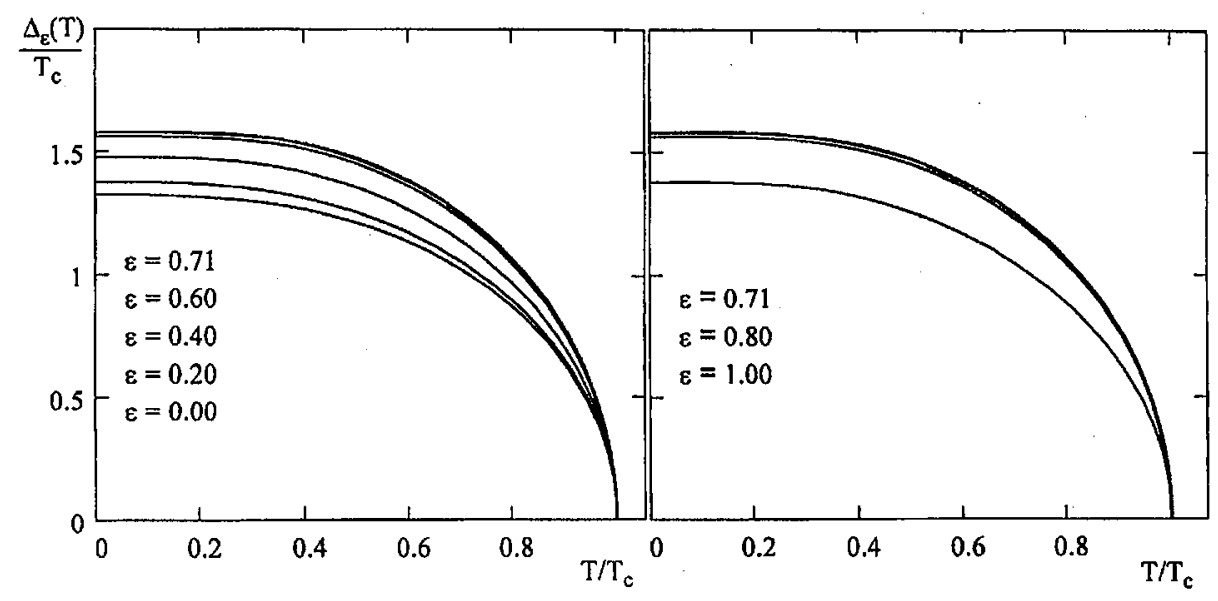

Fig. 2. The reduced energy gaps as a function of reduced temperature obtained numerically according to Eq. (36). The left plot presents the curves which correspond upward to $\varepsilon$ and $\max \left[\Delta_{\varepsilon}(T) / T_{\mathrm{c}}\right]$ according to schematic form: $\left(\varepsilon ; \max \left[\Delta_{\varepsilon}(T) / T_{\mathrm{c}}\right]\right)$ : $(0.00 ; 1.32),(0.10 ; 1.34),(0.20 ; 1.38),(0.40 ; 1.48),(0.60 ; 1.56),(1 / \sqrt{2} ; 1.58)$ - bold line. In the right plot the curves correspond upward to $\varepsilon$ and $\max \left[\Delta_{\varepsilon}(T) / T_{\mathrm{c}}\right]$ according to the same schematic form: $(1.00 ; 1.38),(0.80 ; 1.56),(1 / \sqrt{2} ; 1.58)$ - bold line (compare with Fig. 1).

Let us notice that according to Eq. (29) the heat capacity leap in the limit $T=T_{\mathrm{c}}$ reduces to the form [12]

$$
\Delta C=-\left.2 N(0) \Delta \frac{\partial \Delta}{\partial T}\right|_{T=T_{c}} .
$$

In order to derive the form of the above formula for $D$-paired systems we have to employ Eqs. (4), (10) and (32), then after some transformations we find $\Delta$ as a function temperature for $T \rightarrow T_{\mathrm{c}}$. Taking into account the normal part of the heat capacity [13]

$$
C_{\mathrm{n}}=\frac{2}{3} \pi^{2} N(0) T=\frac{2}{3} \pi^{2} N(0) T_{\mathrm{c}} X(\tau)
$$

we obtain finally

$$
\frac{C_{\mathrm{s}}(T)}{C_{\mathrm{n}}\left(T_{\mathrm{c}}\right)}=1+\frac{12}{7 \zeta(3)\left\langle|D|^{4}\right\rangle}
$$

where, in our case

$$
\left\langle|D|^{4}\right\rangle=\frac{5}{7}\left(4 \varepsilon^{4}-4 \varepsilon^{2}+3\right)
$$

fulfills the conditions: $1.429 \leq\left\langle|D|^{4}\right\rangle \leq 2.143$ and $\left\langle|D|^{4}\right\rangle=1.429$ for $\varepsilon=1 / \sqrt{2}$ and $\left\langle|D|^{4}\right\rangle=2.143$ for $\varepsilon=0$ or $\varepsilon=1$, which ensure the reduced form of heat capacity in relation to its normal value at $T=T_{\mathrm{c}}$ is always smaller for $D$-wave systems than for the BCS state (where $\left\langle|D|^{4}\right\rangle=1$ ). Using the general paramagnetic form of $\Delta C=C_{\mathrm{s}}-C_{\mathrm{n}}$ from Eq. (27) and the function $Y(\tau)$ (Eq. (24)), as well 


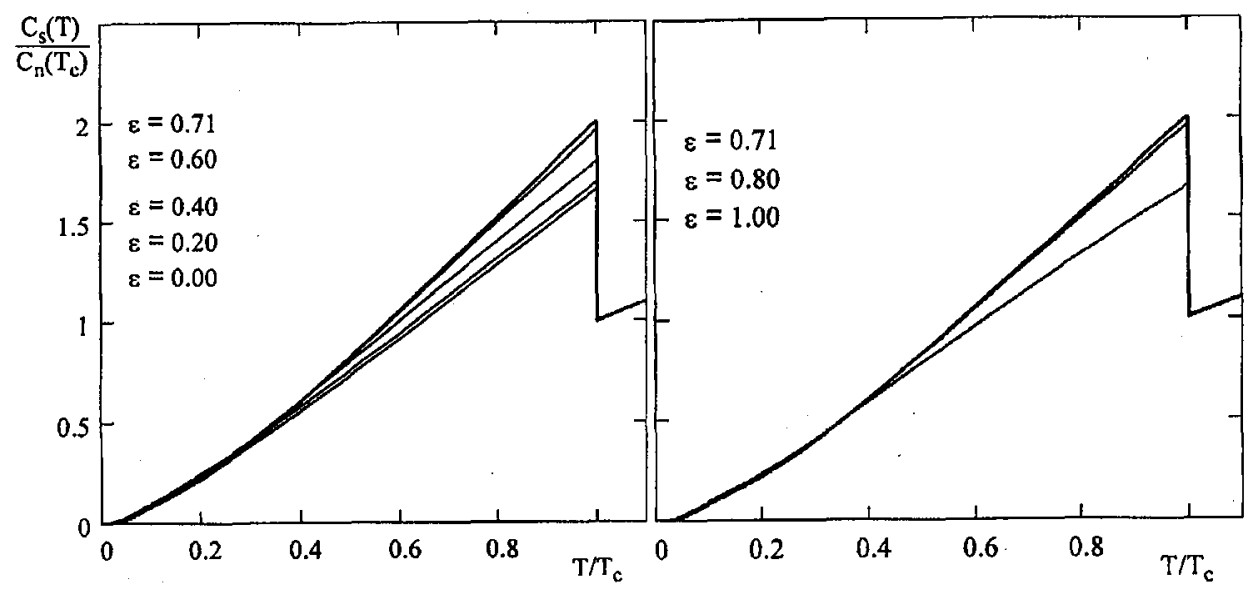

Fig. 3. The plots of the reduced heat capacity $C_{\mathrm{s}}$ of $D$-wave superconductor as a function of reduced temperature calculated numerically according to (29) and (24), for considered previously (see description of Fig. 2) values of $\varepsilon$ (for different $D$-wave paired states). The curves in the left plot correspond upward to $\varepsilon=0, \varepsilon=0.2, \varepsilon=0.4$, $\varepsilon=0.6, \varepsilon=1 / \sqrt{2}$, respectively, and for $T=T_{\mathrm{c}}$ they achieve values: $1.666,1.701,1.811$, 1.961, and 1.998. In the right figure the curves correspond upward to $\varepsilon=1, \varepsilon=0.8$, and $\varepsilon=1 / \sqrt{2}$, respectively, and for $T=T_{c}$ they achieve values: $1.666,1.961,1.998$. The set of $\varepsilon$ is the same as in Fig. 2 for comparison. Note that for $0.3 \leq T / T_{c} \leq 0.4$, the above curves intersect each others and below $T / T_{\mathrm{c}}=0.3$ they fulfil inverse relations than for large temperatures. Moreover, for $T / T_{c} \leq 0.05$ the heat capacity for all $\varepsilon$ becomes very small (the detailed explanations of these facts are given in the paper).

as $X(\tau)$ (Eq. (37)), we may find $\Delta C$ numerically. Eliminating the normal part of the heat capacity we get the shape of a reduced heat capacity $C_{\mathrm{s}}$ of $D$-wave paired superconductor, as a function of reduced temperature, from 0 to $T_{\mathrm{c}}$. Such forms of $C_{\mathrm{s}}\left(T / T_{\mathrm{c}}\right) / C_{\mathrm{n}}(1)$ for the set of $\varepsilon$ previously chosen, analogously as in Fig. 2, are depicted in Fig. 3. Hence the number of curves in Fig. 3 is the same as in Fig. 2. It can be noted that all obtained curves intersect each others in the region of temperatures $0.3 \leq T / T_{\mathrm{c}} \leq 0.4$, and as a result, in the limit $T \rightarrow 0$ the heat capacity and hence the entropy tend to zero more rapidly if the heat capacity achieves larger values at $T_{\mathrm{c}}$. Moreover, considerations presented in Appendix allow us to state that the temperature dependence of $C_{\mathrm{s}}(T)$ in the limit $T \rightarrow 0$ is different than for BCS systems. One possible, phenomenological explanation seems to be the following: because of the structure of $\hat{\Delta}$ at $T \rightarrow 0$ for some $\varepsilon$, when $\hat{\Delta}$ might be very narrow in some regions of spherical angle space, the normal part of the system dominates, and hence we can observe more extensive contribution of the linear component of the heat capacity, which dominates over the second one, i.e. $2 \Delta \partial \Delta / \partial T$, and always exceeds pure BCS component. Note that $2 \Delta \partial \Delta / \partial T$ component can be a specific function of temperature, quite different than for BCS systems. Afterwards, when $T \rightarrow T_{\mathrm{c}}$ and $\hat{\Delta}$ vanishes, the properties of systems depend on $\varepsilon$ and the heat capacity leap becomes greater if the structure of $\hat{\Delta}$ 
is more homogeneous and $\left\langle|D|^{4}\right\rangle$ is to nearer one. Based on these two facts, we maintain that in the intermediate regions of the plots they must intersect each other. Some other aspects of this problem are discussed also in Conclusions.

We do not insert plots of the thermodynamic potential and entropy differences between $D$-wave paired and normal state, but only postulate that the state for which $\Delta_{\varepsilon}(T)$ reaches its maximum (for $\varepsilon=1 / \sqrt{2}$ ) is preferred for all temperatures from 0 to $T_{\mathrm{c}}$ (see Fig. 1). This is in agreement with the results in [2].

\section{Conclusions}

The above formalism developed for $D$-wave paired Fermi system allow us to evaluate thermodynamic properties of a system based on the form of the energy gap as a function of temperature. Moreover it reveals that the general thermodynamic formulas Eq. (22) and Eq. (26)-(30) are identical as those obtained for $S$-wave paired system in [4-6] and [11].

This fact encourages us to assume that the elaborated formulas are universal for arbitrary BCS-like singlet paired Fermi system and, in general, the characteristic thermodynamic properties of the system are entirely determined by the shape of the energy gap vs. temperature. Such a shape can be taken from experimental data, if we only believe that the system under investigation is BCS-like and quasiparticles are paired in a singlet state.

The forms of the heat capacity $C_{\mathrm{s}}$ obtained for the different $D$-waves paired states, as defined above, confirm results of the developed formalism. We state that for some $D$-wave paired states in the case if $\varepsilon$ tends to 0 or 1 , the linear term of the heat capacity dominates in the $T \rightarrow 0$ region. Such properties of the heat capacity seem to be strange when we compare them to the results for classical BCS system, when we observe an exponential increase. However, now the paired states are not isotropic and hence the expression $\Delta(T)|D| / T$, even for $T$ close to zero, can be small in some regions of spherical angle space. Just these small contributions of $\Delta(T)|D| / T$ are responsible for the linear part of the heat capacity. Furthermore, we note also that such linear terms of the heat capacity should appear always if pairing state is not isotropic or pseudoisotropic, however sometimes they could be difficult to observe in the plot.

The above statement is especially evident after including the results presented in Appendix. According to them the linear term of low temperature heat capacity vanishes only if the following equation is fulfilled:

$$
\int_{0}^{1} \frac{Y\left(X^{\prime}\right) \frac{\partial Y\left(X^{\prime}\right)}{\partial X^{\prime}}}{\left(X^{\prime}\right)^{2}} \mathrm{~d} X^{\prime}=-\frac{1}{3} \pi^{2}
$$

which is true only in the case of the BCS energy gap. Hence, if a shape of an energy gap differs from the BCS one, a linear term of low temperature heat capacity must exist.

\section{Appendix}

We show that after some algebraic transformations one can obtain the general form of the heat capacity difference in the limit $T \rightarrow 0$. 
According to Eq. (29) we have

$$
\frac{\Delta C}{2 N(0) T_{\mathrm{c}} F_{\infty}}=X \int_{X}^{1} \frac{Y^{2}\left(X^{\prime}\right)-Y^{2}(X)}{\left(X^{\prime}\right)^{3}} \mathrm{~d} X^{\prime}-\frac{1}{2} X Y^{2}(X)-Y(X) \frac{\partial Y(X)}{\partial X} .
$$

Now, after integration by parts, we get

$$
\begin{aligned}
& \frac{\Delta C}{2 N(0) T_{\mathrm{c}} F_{\infty}}=X \int_{X}^{1} \frac{Y\left(X^{\prime}\right) \frac{\partial Y\left(X^{\prime}\right)}{\partial X^{\prime}}}{\left(X^{\prime}\right)^{2}} \mathrm{~d} X^{\prime}-Y(X) \frac{\partial Y(X)}{\partial X} \\
& =X \int_{0}^{1} \frac{Y\left(X^{\prime}\right) \frac{\partial Y\left(X^{\prime}\right)}{\partial X^{\prime}}}{\left(X^{\prime}\right)^{2}} \mathrm{~d} X^{\prime}-X \int_{0}^{X} \frac{\frac{\partial Y^{2}\left(X^{\prime}\right)}{\partial\left(X^{\prime}\right){ }^{2}}}{X^{\prime}} \mathrm{d} X^{\prime}-Y(X) \frac{\partial Y(X)}{\partial X} .
\end{aligned}
$$

However, the last form of $\Delta C$ is correct only if the first integral is convergent. Then, using L'Hôspital's rule we obtain

$$
\frac{\Delta C}{2 N(0) T_{\mathrm{c}} \bar{F}_{\infty}}=X \int_{0}^{1} \frac{Y\left(X^{\prime}\right) \frac{\partial Y\left(X^{\prime}\right)}{\partial X^{\prime}}}{\left(X^{\prime}\right)^{2}} d X^{\prime}-2 Y(X) \frac{\partial Y(X)}{\partial X}
$$

where the other terms must vanish if the integral is to be convergent.

The last formula proves that $\Delta C$ can be always performed in the form of Eq. (31).

\section{References}

[1] D. Scalapino, Phys. Rep. 250, 329 (1995).

[2] P.W. Anderson, P. Morel, Phys. Rev. 123, 1911 (1961).

[3] R. Gonczarek, M. Mulak, Acta Phys. Pol. A 86, 1007 (1994).

[4] M. Mulak, R. Gonczarek, Acta Phys. Pol. A 89, 689 (1996).

[5] M. Mulak, R. Gonczarek, Czech. J. Phys. 46, S6 3379 (1996).

[6] M. Mulak, R. Gonczarek, Acta Phys. Pol. A 92, 1177 (1997).

[7] W. Kumala, R. Gonczarek, Acta Phys. Pol. A 93, 539 (1998).

[8] J. Spałek, Phys. Rev. B 40, 5180 (1989).

[9] J. Czerwonko, Physica $C$ 235-240, 2337 (1994).

[10] J. Maćkowiak, P. Tarasewicz, Mol. Phys. Rep. 15/16, 61 (1996).

[11] R. Gonczarek, M. Mulak, Physica Status Solidi B 208, 457 (1998).

[12] A.L. Fetter, J.D. Walecka, Quantum Theory of Many-Particle Systems, McGraw-Hill Book Co., New York 1971.

[13] R. Gonczarek, M. Mulak, submitted to Phys. Lett. A. 